Lepr Rev (2000) 71, 325-331

\title{
Relapses after fixed duration multiple drug therapy: the AMFES cohort
}

\author{
SHIBRU GEBRE*, PAUL SAUNDERSON* \& \\ PETER BYASS** \\ *ALERT, PO Box 165, Addis Ababa, Ethiopia \\ **School of Community Health Science, Nottingham University, \\ Nottingham, UK and Department of Public Health and Clinical \\ Medicine, Umeå University, Umeå, Sweden
}

Accepted for publication 16 June 2000

\begin{abstract}
Summary Relapse rates after multiple-drug therapy (MDT) have been low, although there remains a concern about the possibility of late relapse in those with an initially high bacterial load. In all, 502 patients in the AMFES cohort completed fixedduration MDT and are included in this report. There have been no confirmed relapses in the AMFES cohort, in a follow-up period of up to 8 years after completion of treatment, even in the 57 cases with an initial average bacillary index of $\geq 4.0,20$ of whom have been followed for more than 5 years after ceasing MDT. Methods of diagnosing a relapse are discussed.
\end{abstract}

\section{Introduction}

Relapse rates after multiple-drug therapy (MDT) for leprosy are widely reported to be low, ${ }^{1}$ to the extent that the recommended duration of treatment for multibacillary (MB) cases has been reduced to 12 months. $^{2}$

Making the diagnosis of relapse is not necessarily straightforward, and there is a possibility of overdiagnosis. For paucibacillary (PB) cases reversal reactions can be confused with relapse and there is no gold standard for comparison, as the organism cannot be isolated in these cases. In a review of over 40,000 PB cases released from treatment in India, $0.29 \%$ relapsed according to clinical criteria, but it was noted that it was likely that most of these cases recorded as relapses were late reversal reactions. ${ }^{3}$ This is confirmed in a study from Ethiopia, where less than half of the $1.1 \%$ of PB cases who were recorded as having relapsed were thought to be true relapses on review. ${ }^{4}$ Histological examination is often unable to distinguish between a reaction and a PB relapse. ${ }^{4,5} \mathrm{~A}$ therapeutic trial with steroids is advocated as a means of making a clear diagnosis. ${ }^{6}$

Amongst MB patients reversal reactions may also be confused with relapse, but the

Correspondence to: P. Saunderson, ALM, 1 ALM Way, Greenville SC 29601, USA (e-mail: psaunderson@ leprosy.org) 
possibility of confirming a relapse bacteriologically is available, either by skin smears to show a rise in bacillary index (BI) and/or a positive morphological index (MI), histology (using a stain for acid-fast bacilli) or by mouse-foot-pad inoculation. In the study in Ethiopia quoted above, $1.0 \%$ of $\mathrm{MB}$ cases were recorded as having relapsed, but none of these was confirmed on closer scrutiny: a common problem was to diagnose a relapse when only one skin smear result showed a rise in BI. Guidelines for the programme required a second smear to confirm the rise in $\mathrm{BI}$, or an $\mathrm{MI}$ of $2 \%$ or more. $^{4}$

Relapses are usually reported within 2-3 years after release from treatment (RFT), when reversal reactions are also more likely to occur. This is partly for logistic reasons with few studies being able to follow patients for long periods. In one long-term study of rifampicincontaining but non-standard multidrug regimens, relapses occurred in two groups: the majority had early relapses, before 3.5 years after release from treatment (median 22 months), while the remainder had late relapses more than 3.5 years after release from treatment (median 5 years). ${ }^{7}$ It was suggested that the early relapses were likely to be due to insufficient treatment and the late relapses to persisting organisms.

A more recent long-term study showed a surprisingly high rate of relapse in those with an initial average BI of $\geq 4$, using fixed-duration WHO-MDT; late relapses occurred in seven $(20 \%)$ of these patients at 5 years $\left( \pm 2\right.$ years) after release from treatment. ${ }^{8}$ The relapses had an increased BI and new skin lesions, and were confirmed in four cases by growth in the mouse-foot-pad and in six cases by a positive MI.

\section{Materials and methods}

The ALERT MDT Field Evaluation Study (AMFES) recruited 660 patients between 1988 and 1993. There were 10 exclusions due to incorrect diagnosis or incorrect enrolment procedures. Of the 650 patients included, 56 were relapse cases after dapsone monotherapy and 594 were new cases. All were treated with fixed-duration MDT. A total of $502(77 \%)$ of the 650 patients completed treatment and are included in the relapse study. Patients were reviewed every 6 months after release from treatment, although there was quite a large dropout immediately after stopping MDT. Random samples of urine were tested for dapsone during both treatment and surveillance.

Patients attending a 6-monthly review were examined clinically for signs of new or enlarging skin lesions and for signs of nerve function impairment (NFI). In MB cases a skin smear was routinely done each year, at four standard sites and from any suspect skin lesion. A relapse could be suspected because of findings in the skin, or in the skin smear results. The late appearance of reactions or nerve function impairment was also considered as a sign of possible relapse. For the purposes of this paper, all patients who were given steroids (indicating a severe reaction or NFI) more than 3 years after the diagnosis of leprosy had been made were defined as possible relapses.

Skin smears were all read at the ALERT Hospital laboratory, where smears were kept for review purposes for 1 year only. Smears were fixed and stained by the Ziehl-Neelsen method and examined using a binocular light microscope with an oil immersion lens at $\times 100$ magnification. The BI was reported for all four sites, so that although the BI was normally taken to be the highest reading, an average can be calculated for each test. The MI was taken as the percentage of solid staining bacilli in 100 examined.

The AMFES protocol clearly defined the management of suspected relapses. In summary, 
an $\mathrm{MB}$ relapse would be indicated by a skin smear after release from treatment with an MI of at least $2 \%$, or two skin smears in which the highest BI is 2 units above a previously recorded level. A PB relapse would be confirmed by the appearance of new lesions or the extension of existing lesions, after a course of steroids. If the diagnosis of a relapse is uncertain, as is commonly the case, patients should be treated with steroids (without anti-leprosy treatment) and reviewed within a period of 6 months for PB cases and 12 months for MB cases. If the changes in the skin resolve during this observation period, the likelihood of a relapse is reduced, while if the lesions remain or increase, a relapse is confirmed. Histological examinations should be carried out for all suspected relapses, but the results were not to be taken into account in reaching a clinical conclusion: these results would be used later to assess the correlation between histology and the clinical findings. The mouse footpad assay should be used for suspected MB relapses and would allow subsequent investigation for possible drug resistance.

Patients who were prescribed steroids for reactions and nerve function impairment after they had completed MDT were not given additional anti-leprosy drugs.

\section{Results}

Table 1 shows the length of follow-up of AMFES patients, including those with an initially high BI who may be considered at greatest risk of relapse. In all, 12/677 (1.8\%) random urine specimens tested positive for dapsone during the surveillance period.

There have been no confirmed relapses in the AMFES cohort, although one patient was re-treated by another doctor on the basis of clinical suspicion alone. Relapse was suspected at some time in $40(6 \%)$ patients and Table 2 shows the composition of this group.

New lesions in PB cases were usually noticed by the patient and presented to the health worker. In all four cases, these new lesions were thought by health staff to be on the same sites as previous lesions, but patients were unhappy not to be re-treated. One patient was thought by one doctor to have relapsed and by another to have had a reversal reaction and unfortunately this patient was re-treated without going through the correct AMFES procedures (namely, treatment with steroids alone in the first instance and re-assessment). The others were treated with steroids (two) or observed (one), with good results. One patient has had four biopsies over a 5-year period, all indicating varying degrees of delayed type hypersensitivity (DTH) in tuberculoid leprosy without any AFB; steroids have been helpful

Table 1. Follow-up after the end of MDT of AMFES patients who completed treatment

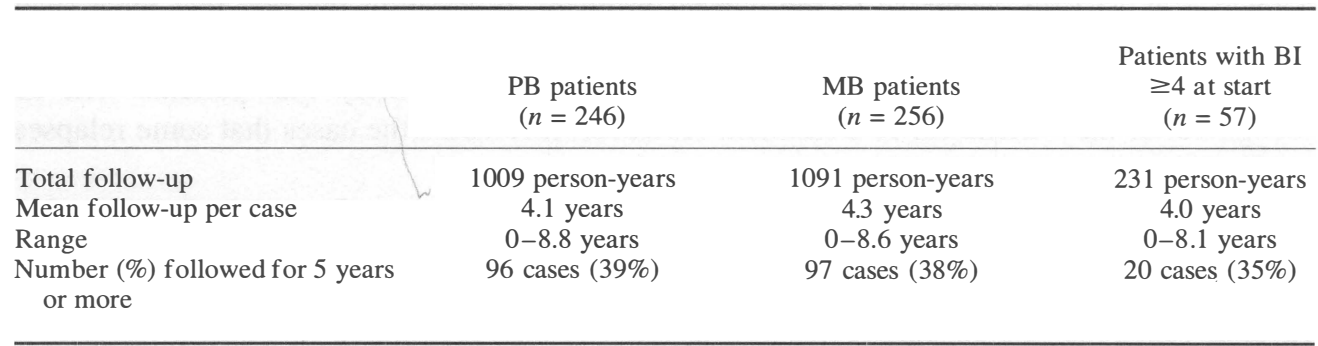


Table 2. Reasons for suspecting a relapse in 40 patients

\begin{tabular}{lcc} 
Reason for suspecting a relapse & Number of cases & Timing of suspicious events \\
Possible new lesions & $6(2 \mathrm{MB}, 4 \mathrm{~PB})$ & $2-5$ years after RFT \\
Bacteriological results & $7(6 \mathrm{MB}, 1 \mathrm{~PB})$ & Median 4 years \\
Late reactions/neuritis & $27(19 \mathrm{MB}, 8 \mathrm{~PB})$ & Variable \\
Total & $40(27 \mathrm{MB}, 13 \mathrm{~PB})$ & \\
\hline
\end{tabular}

on several occasions in this case, although the patient has continued to complain of the skin lesions, of ten insisting that they were new.

One of the MB cases with new lesions had a BI of 0 and was treated as a case of reversal reaction with good results. The other $\mathrm{MB}$ case had persisting fleshy nodules on the ear lobes with a slowly declining BI; the BI gradually settled to zero and a mouse foot-pad test (3 years after release from treatment) showed no growth.

The bacteriological suspects included one PB patient who had a nerve biopsy 10 months after release from treatment to define the nature of a reaction; one AFB was seen in the biopsy slide, but this was taken to be acceptable and the patient was not re-treated. Of the MB cases with suspicious bacteriological results, two were thought to be administrative errors as the BI rose from $0 / 1$ to 4 and then returned to zero a few months later, remaining negative ever since; one of these had a mouse footpad assay done, which was negative. Three cases showed a slight rise in BI 4 years after release from treatment that was reversed in subsequent smears. One case remained with a persistently high BI (a reading at one site of at least 4 ) for 5 years and had recurrent neuritis and ENL; a mouse footpad assay was negative and smears became negative at 8 years, with no nerve function impairment at that time. The MI was never positive in any patient after release from treatment.

The 27 cases with late or prolonged leprosy reactions/neuritis were treated for their reactions and observed for any other signs of relapse; regular smears were done. No further signs of relapse were noted in any of them.

\section{Discussion}

The rate of confirmed relapses in the AMFES cohort is zero. While the number of patients lost to follow-up is significant (only $38 \%$ have completed 5 years surveillance), it is likely that any relapses in this group would have come to the attention of the staff involved in AMFES. Patients who move away from home often come to Addis Ababa and are likely to come to ALERT if they develop lesions suggesting a relapse. One patient was treated as a relapse at ALERT, contrary to the correct protocol, illustrating the fact that most such cases will attend ALERT eventually, occasionally denying their previous treatment. The very low turnover of staff involved in AMFES, makes it likely that patients will be recognized if they attend for re-treatment. However, it remains the cases that some relapses could have been missed by this study.

The appearance of new lesions in PB patients occurred most frequently 2 years after release from treatment and can be regarded as a manifestation of a reversal reaction. Most of the suspected MB relapses occurred at 4-5 years after release from treatment and were either reversal reactions (the appearance of new lesions) or unsubstantiated skin smear results. 
PB relapses after MB leprosy have been reported, ${ }^{9,10}$ but were not suspected in this cohort. MB relapses after PB leprosy are generally thought to be due to inadequate treatment because of misclassification at diagnosis; ${ }^{4}$ although the definition of PB leprosy in this cohort is wide, classification was done with great care, so that all except three patients with positive smears got the $\mathrm{MB}$ regimen and there have been no suspected relapses in this category.

The major current concern is the possibility of relapse after 5 years in the group of patients with an initially high BI. ${ }^{11-14}$ Many previous reports of low relapse rates in MB patients after MDT may be unreliable, as they included patients who had had many years of dapsone monotherapy and therefore had a low bacterial load at the start of MDT, or had MDT for more than 2 years, until smear negativity. ${ }^{11}$

In the AMFES study, 57 patients had an average BI of $\geq 4$ at the start of MDT (four were true relapses after dapsone monotherapy) and were given fixed-duration treatment for 2 years. It has been suggested that in most programmes this will be a small group as compared with the overall leprosy burden, ${ }^{2}$ but in this cohort it is $9 \%$ of the total case load, which is not insignificant. However, 20 patients in this group have been followed for more than 5 years, without a confirmed relapse, suggesting that the high relapse rates some years after fixedduration MDT found in Mali $^{8}$ and elsewhere ${ }^{12}$ may not be typical.

Reversal reactions and neuritis can occur at any time during the 5 years after release from treatment in PB patients, although they certainly become less common over that period, as shown in accompanying papers from AMFES. In such cases, management with steroids, especially if nerve function impairment is present is most important. The diagnosis of relapse is not urgent and can be made after a trial of steroids. Skin smears or a biopsy stained for AFB will indicate a multibacillary relapse.

In $\mathrm{MB}$ patients, reversal reactions and neuritis may also occur at any time in the 5 years after release from treatment. Suspected relapses require at least two skin smears to show a rise in $\mathrm{BI}$ of more than $1 \log$ unit, or to show an MI of at least $2 \%$. In many programmes, the number of skin smears being done is greatly reduced, either to zero or to one at diagnosis, for technical and logistic reasons. In such situations a smear taken some years after release from treatment will be difficult to interpret, as rates of decline in BI vary quite widely. Some indication of the viability of the organisms found on the smear would be valuable.

The MI has been used as a method of assessing the efficacy of treatment regimens in leprosy. There are other methods of assessing the viability of mycobacteria in the tissues that are more sensitive, but they are much less applicable in leprosy endemic areas. ${ }^{15}$ In one study the MI was positive in 59/68 specimens with viable bacilli, giving a sensitivity of $87 \%$, and was $100 \%$ specific, in that an MI of $1 \%$ or more always indicated viable organisms, although testing did not include the mouse footpad assay. ${ }^{16}$ Previous studies have shown, however, that the measurement of the MI is difficult to standardize and unreliable under field conditions. ${ }^{17,18}$ The MI could become a useful indicator of MB relapse in future, but a well-functioning reference laboratory would be the only appropriate place for this to be done. It is suggested that positive skin smears from suspected relapses should be sent to a reference laboratory for estimation of the MI.

If skin smears cannot be taken and read at all, the diagnosis of relapse will depend on the clinical findings alone. The clinical experience required to suspect and diagnose a relapse is, however, also disappearing. In testing a number of different regimens, the Marchoux Chemotherapy Study Group have seen many multibacillary relapses in which the new lesions are mainly nodules and lepromas. ${ }^{19}$ In view of the high acceptability of MDT, it may be 
appropriate to advocate a low threshold for the diagnosis of relapse, based on the definite appearance of new lesions, in particular those that are nodular in form.

Steroids have been widely used in this study after completion of MDT without relapse, indicating that cover with anti-leprosy drugs is not required in this situation.

In summary, while relapse must always be considered, the reappearance of skin lesions in leprosy patients in the 5 years after release from treatment is likely to be due to a reversal reaction and should be treated as such, especially if there is accompanying nerve involvement. Definitely new lesions, especially if nodular in form, are more likely to be due to a relapse.

Skin smears should be done if possible, and if positive, further steps should be taken to identify true multibacillary relapses:

1. Review any previous smear results.

2. Refer the smear for measurement of the MI, if possible.

3. Biopsy an active lesion, one section being stained for acid-fast bacilli.

After collecting this evidence and reviewing the results of anti-reaction treatment, an informed decision can be made as to the need for a further course of MDT.

While no definitive conclusion can be made about the sub-group of patients with a high initial BI, the AMFES results suggest that 2 years of WHO-MDT is a satisfactory treatment regimen. It is proposed to follow this sub-group for a further 5 years.

\section{Acknowledgements}

We thank the staff of the ALERT Leprosy/TB Control Division for their dedication and perseverance in managing the patients and collecting data over so many years. The financial support of ILEP, through Netherlands Leprosy Relief (NLR), has been constant throughout the 12 years of the study and is gratefully acknowledged. We also thank ALERT as a whole for institutional and administrative support, and Professor Sebastian Lucas for reviewing certain histological specimens.

\section{References}

1 WHO Leprosy Unit. Risk of relapse in leprosy. WHO document 1994; WHO/CTD/LEP/94.1.

2 Ji B. Why multidrug therapy for multibacillary leprosy can be shortened to 12 months. Lepr Rev, 1998; 69: 106109.

${ }^{3}$ Lobo D. Treatment failures with multidrug therapy. Lepr Rev, 1992; 63: 93s-98s.

${ }^{4}$ Becx-Bleumink M. Relapses among leprosy patients treated with multidrug therapy: experience in the leprosy control program of the All Africa Leprosy and Rehabilitation Training Center (ALERT) in Ethiopia; practical difficulties with diagnosing relapses; operational procedures and criteria for diagnosing relapses. Int J Lepr, 1992; 60: $421-435$.

5 Pattyn SR, Husser JA, Baquillon G et al. Evaluation of five treatment regimens, using either dapsone monotherapy or several doses of rifampicin in the treatment of paucibacillary leprosy. Lepr Rev, 1990; 61: 151-156.

${ }^{6}$ Ramachandran A, Seshadri PS. Relapse or reversal reaction: the case for a therapeutic trial of steroids. Lepr Rev, 1988; 59: 271-272.

7 Pattyn SR, Groenen G, Bourland $\mathbf{J}$ et al. The incubation time of relapses after treatment of multibacillary leprosy with rifampicin containing regimens. Eur J Epidemiol, 1988; 4: 231-234.

${ }^{8}$ Jamet P, Ji B, Marchoux Chemotherapy Group. Relapse after long-term follow-up of multibacillary patients treated by WHO multidrug regimen. Int J Lepr, 1995; 63: 195-201.

9 Waters MF, Ridley DS. Tuberculoid relapse in lepromatous leprosy. Lepr Rev, 1990; 61: 353-365.

10 Saunderson PR, Waddell KM. Tuberculoid relapse in lepromatous leprosy (Letter). Lepr Rev, 1993; 64: $170-171$. 
11 Waters MF. Relapse following various types of multidrug therapy in multibacillary leprosy (Editorial). Lepr Rev, 1995; 66: 1-9.

12 Waters MF. Is it safe to shorten multidrug therapy for lepromatous (LL and BL) leprosy to 12 months? Lepr Rev, 1998; 69: 110-111.

13 Lynch P. Multidrug therapy. Lepr Rev, 1999; 70: 70-71.

14 van Brakel WH. Proposal regarding MB MDT. Lepr Rev, 1999; 70: 71-73.

15 Odinsen O, Nilson T, Humber DP. Viability of Mycobacterium leprae: a comparison of morphological index and fluorescent staining techniques in slit-skin smears and M. leprae suspensions. Int J Lepr, 1986; 54: 403-408.

16 Katoch VM, Katoch K, Ramanathan U et al. Effect of chemotherapy on viability of Mycobacterium leprae as determined by ATP content, morphological index and FDA-EB fluorescent staining. Int J Lepr, 1989; 57: 615621.

17 McRae DH, Shepard CC. Relationship between the staining quality of Mycobacterium leprae and inf ectivity for mice. Infect Immun, 1971; 3: 116-120.

18 Shepard CC, McRae DH. Mycobacterium leprae in mice: minimal infectious dose, relationship between staining quality and infectivity, and effect of cortisone. J Bacteriol, 1965; 89: 365-372.

19 Marchoux Chemotherapy Group. Relapses in multibacillary leprosy patients after stopping treatment with rifampicin-containing combined regimens. Int J Lepr, 1992; 60: 525-535. 